\title{
Tomografia elétrica 2D e gravimetria para detecção de sítio contaminado por derivados de petróleo em área de passivo ambiental
}

\author{
$2 D$ electrical tomography and gravimetry for the detection of a \\ site contaminated by oil derivates in an area of environmental liability
}

\section{Valter Antonio Becegato', Francisco José Fonseca Ferreira², Rodoilton Stevanato³, Luís Gustavo de Castro ${ }^{4}$, Jefferson Ulisses da Cunha ${ }^{5}$, Vitor Rodolfo Becegato ${ }^{6}$}

\begin{abstract}
RESUMO
Foram realizados ensaios geofísicos, como caminhamento elétrico (arranjo dipolo-dipolo) com $\mathrm{AB}=\mathrm{MN}=4 \mathrm{~m}$, totalizando 1.024 m de perfis, e levantamento gravimétrico, totalizando 273 estações separadas de 4 em 4 m. Os valores máximos, mínimo, médio e desvio padrão da resistividade elétrica real em ohm.m foram, respectivamente: 35, 2.276, 432 e 344. Com relação às anomalias gravimétricas Bouguer, observou-se que a porção oriental é caracterizada por sinais com maiores amplitudes, em contraposição ao segmento ocidental, onde as mesmas são intermediárias a baixas, destacando-se valores mínimos entre as estações O-44 m das linhas L4, L5 e L6. Detectou-se a presença de hidrocarboneto a $3 \mathrm{~m}$ de profundidade, nas linhas L8 e L9. Análises laboratoriais das amostras de solos e água apontaram valores PHAs que excedem os parâmetros estabelecidos pela resolução CONAMA no 357/2005.
\end{abstract}

Palavras-chave: passivo ambiental; petróleo; tomografia elétrica; gravimetria.

\begin{abstract}
The following geophysical essays were performed: DC resistivity survey (dipole-dipole array) with $\mathrm{AB}=\mathrm{MN}=4 \mathrm{~m}$, totaling $1.024 \mathrm{~m}$ of profiles, and a magnetometric survey, totaling 273 stations split every $4 \mathrm{~m}$. The maximum, minimum, and average values, and the standard deviation of the actual resistivity in ohm.m were respectively: 35, 2,276, 432, and 344. Regarding the Bouguer gravity anomalies, it was observed that the eastern portion is characterized by signals with larger amplitudes, in contrast to the western segment, where these are intermediate to low, especially minimum values between stations 0-44 meters, from lines L4, L5 and L6. The presence of hydrocarbons was detected at a depth of $3 \mathrm{~m}$ in lines L8 and L9. Laboratory analysis of soil and water samples showed values of PHAs that exceed the parameters established by the CONAMA resolution no 357/2005.
\end{abstract}

Keywords: environmental liability; petroleum; electrical tomography; gravimetry.

\section{INTRODUÇÃO}

Os solos são derivados principalmente dos diferentes tipos de rochas, cuja gênese dos mesmos se deve aos fatores de formação. Isso, consequentemente, leva a um comportamento diferenciado da condução da corrente elétrica em diferentes tipos de solos (YOON \& PARK, 2001; BECEGATO \& FERREIRA, 2005; SAMOUËLIAN et al., 2005) - como os arenosos e argilosos - bem como à presença de água, substâncias metálicas, sais e outros solutos presentes nos poros dos solos, o que interfere na resistividade elétrica.

Diversos autores (FRIEDEL et al., 2006; AMATO et al., 2009; BECEGATO et al., 2009; SÉGER et al., 2009; SUDHA et al., 2009; HADZICK et al., 2011; USTRA et al., 2012) utilizam a tomografia elétrica em diferentes estudos, por se tratar de um método rápido, não invasivo e com respostas confiáveis.

Trabalho desenvolvido na Universidade Federal do Paraná (UFPR) - Curitiba (PR), Brasil.

'Doutor em Geologia Ambiental pela Universidade Federal do Paraná (UFPR). Professor Associado do Departamento de Engenharia Ambiental da Universidade do Estado de Santa Catarina (UDESC) - Lages (SC), Brasil.

2Doutor em Geologia pela Universidade de São Paulo. Professor Associado do Departamento de Geologia da Universidade Federal do Paraná (UFPR) - Curitiba (PR), Brasil. ${ }^{3}$ Mestre em Geologia pela Universidade Federal do Paraná. Geólogo no Laboratório de Geofísica Aplicada (LPGA) da UFPR - Curitiba (PR), Brasil.

${ }^{4}$ Doutorando em Geologia pela Universidade Federal do Paraná. Geólogo no Laboratório de Geofísica Aplicada (LPGA) da UFPR - Curitiba (PR), Brasil.

${ }^{5}$ Mestre em Geologia Ambiental pela Universidade Federal do Paraná. Engenheiro cartógrafo pelo Laboratório de Geofísica Aplicada (LPGA) da UFPR - Curitiba (PR), Brasil. ${ }^{6}$ Mestrando em Ciências Ambientais pela Universidade do Estado de Santa Catarina (UDESC) - Lages (SC), Brasil.

Endereço para correspondência: Valter Antonio Becegato - Universidade do Estado de Santa Catarina (UDESC) - Avenida Luis de Camões, 2090 - 88.520.000 - Lages (SC),

Brasil - valter.becegato@udesc.br

Recebido: 21/03/13 - Aceito: 25/07/16 - Reg. ABES: 99442 
Passivos ambientais - como os lixões, os cemitérios horizontais e a contaminação por hidrocarbonetos em solos e lençol freático - podem ser estudados e monitorados com a utilização de métodos geofísicos, entre os quais a tomografia elétrica (ATEKWANA et al., 2000; BERNSTONE et al., 2000; DE LA VEGA et al., 2003; BRAGA \& CARDINALI, 2005; MENEZES et al., 2011; BERTOLLA et al., 2014).

A resistividade elétrica, largamente utilizada em estudos do meio ambiente, é considerada uma poderosa ferramenta para diagnosticar estudos de subsolo, geológicos e geotécnicos, conforme pode ser constatado em alguns trabalhos como os de Sauck (2000), Martinho et al. (2006), Lago et al. (2009), Rosales et al. (2012) e Bortolozo et al. (2014).

O presente trabalho mostra os resultados de ensaios geofísicos realizados em uma área de passivo ambiental, cujo objetivo foi localizar em subsuperfície, rejeitos de produtos derivados de hidrocarbonetos, os quais estavam contaminando um lago situado nas proximidades de uma distribuidora de petróleo. Produziram-se 13 linhas de caminhamento elétrico, arranjo dipolo-dipolo com $\mathrm{AB}=\mathrm{MN}=4 \mathrm{~m}$, totalizando $1.024 \mathrm{~m}$ de perfis. Para a aquisição dos dados gravimétricos, obtiveram-se dados de 273 estações separadas de 4 em 4 m.

\section{Localização e descrição da área}

A área localiza-se (Figura 1) no município de Renascença, Estado do Paraná, Terceiro Planalto Paranaense, cuja constituição geológica é representada pelas rochas do tipo basalto toleítico com intercalações de arenito e rochas vulcânicas ácidas, ambas cortadas por diques e sills de diabásio da formação Serra Geral do Grupo São Bento. Os solos predominantes são do grupo dos Latossolos.

Ao lado da área objeto deste trabalho, existe uma distribuidora de derivados de petróleo, cujo proprietário também é o dono da área da presente pesquisa. Como foi detectada a presença de hidrocarbonetos junto à margem de um espelho d'água, a distribuidora foi fiscalizada pelo Instituto Ambiental do Paraná, por entender que poderia haver vazamentos nos

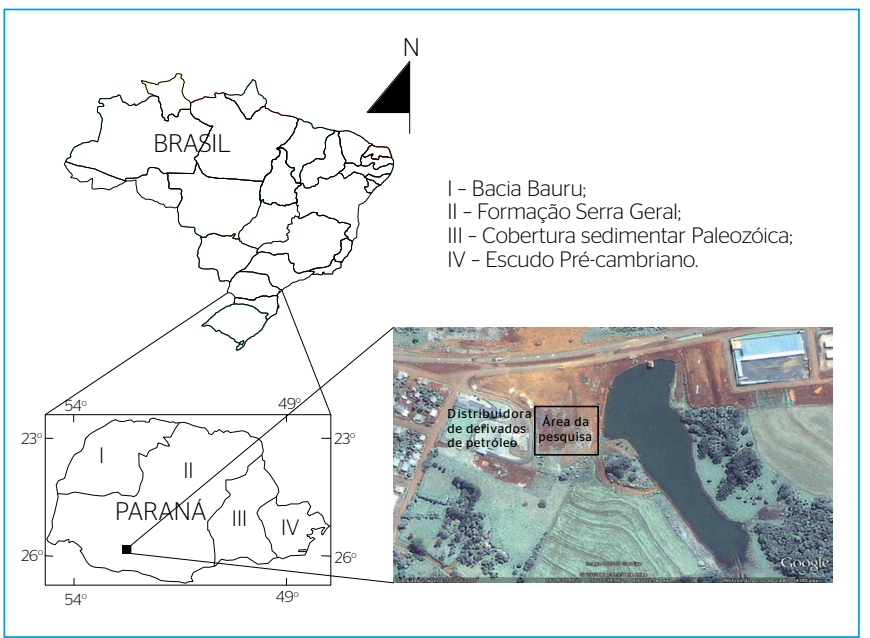

Figura 1 - Localização da área da pesquisa. tanques de armazenamento de combustíveis. Porém, depois das pesquisas na área dos tanques de armazenamento, não foi constatado nada irregular.

Após estudos sobre a ocupação da área, que ocorreu anteriormente à instalação da distribuidora, constatou-se que o local foi utilizado nos anos 1970 como um depósito de descarte de produtos derivados de petróleo por empreiteiras que construíram a BR-280. Tal espaço era um pátio de máquinas, onde eram realizados troca de óleo das máquinas, lavação, jateamento, entre outros procedimentos. Nesse contexto, utilizaram-se métodos geofísicos para encontrar a célula onde o sítio contaminado estaria localizado.

\section{METODOLOGIA}

Efetuou-se a demarcação da área para instalação das linhas e dos pontos por GPS, a fim de se obter os dados georreferenciados. Foram localizadas 13 linhas para ambos os métodos. Tais ensaios envolveram a aplicação dos métodos gravimétrico, com 273 estações separadas de 4 em 4 m, e resistivimétrico estabelecendo-se 13 linhas de caminhamento elétrico, arranjo dipolo-dipolo com $\mathrm{AB}=\mathrm{MN}=4 \mathrm{~m}$, totalizando $1.024 \mathrm{~m}$ de perfis.

\section{Arranjo dipolo-dipolo (imageamento elétrico 2-D)}

A eletrorresistividade foi definida a partir do conceito fundamental da Lei de Ohm, a qual estabelece uma relação empírica entre a corrente elétrica fluindo através de um condutor e o potencial de voltagem requerido para conduzir essa corrente. Essa lei conclui que a corrente (I) é proporcional à voltagem $(V)$ e é dada pela seguinte expressão: $V=R . I$, onde $R$ é a resistência elétrica dos materiais e sua unidade é ohm (W).

Quando o objetivo em estudos ambientais é saber como se comporta a corrente elétrica nos diferentes meios, injeta-se uma corrente contínua I no terreno utilizando dois eletrodos (A e B), e a diferença de potencial DV é medida por meio de outros dois eletrodos ( $\mathrm{M} \mathrm{e} \mathrm{N}$ ). A função resistividade aparente $\left(r_{a}\right)$ é dada pela seguinte expressão: $\mathrm{r}_{\mathrm{a}}=\mathrm{K}^{\star} \mathrm{DV} / \mathrm{I}$. O fator K que multiplica DV/I é puramente geométrico e depende apenas da disposição dos eletrodos A, B, M e N. A resistividade aparente $\left(\mathrm{r}_{\mathrm{a}}\right)$ não é um parâmetro físico do meio, mas um efeito integrado sobre um segmento do semiespaço, para a qual contribuem os valores da resistividade em cada ponto, a geometria elétrica do terreno e a disposição geométrica dos eletrodos. Conhecendo-se a corrente que percorre o subsolo, a disposição dos eletrodos e a diferença de potencial, pode-se calcular o valor da resistividade aparente em cada posição do arranjo (Figura 2).

\section{Gravimetria}

As principais correções aplicadas aos dados do levantamento de campo para os dados gravimétricos foram: maré e deriva instrumental. A primeira foi processada no programa interno do equipamento para cada 
ponto, e a segunda calculada com o auxílio de uma planilha eletrônica. Além disso, os valores finais de gravidade, para cada estação, foram corrigidos quanto aos efeitos citados. A partir desses valores, foram realizadas as demais correções (Equação 1), que consistem na redução dos valores de gravidade à superfície do geóide, a saber:

Correção de Latitude: $\mathrm{GI}=978032,67714^{*}\{[1+0,00193185138639 *$ $\left.\left(\operatorname{sen}^{2} j\right)\right] /\left[\left(1-0,00669437999013^{\star}\left(\operatorname{sen}^{2} j\right)\right]^{1 / 2}\right\}$, onde GI é a gravidade teórica em mGal (correção de latitude) e j é a latitude;

Correção Ar Livre: $\mathrm{C}_{\mathrm{AL}}=\left[0,308767763-0,000439834^{\star}(\operatorname{sen} 2 \mathrm{j})-\right.$ $\left.0,000000072124602^{*} \mathrm{~h}\right]^{*} \mathrm{~h}, \mathrm{em} \mathrm{mGal}$, onde h é a altitude e j é a latitude;

Correção Bouguer: $C_{B}=0,04198088^{*}$ rh-B, em mGal, onde $r$ $\left(2,67 \mathrm{~g} / \mathrm{cm}^{3}\right)$ é a densidade crustal média, h é a altitude e B é a correção de curvatura. Esta última correção equivale à conversão da correção Bouguer, de um platô infinito para uma calota esférica, cuja espessura é a elevação da estação com raio de 166,735 km a partir da mencionada estação;

Correção de Terreno: a correção de terreno $\left(C_{T}\right)$ foi realizada a partir da aplicação automática simultânea da carta de Hammer aos pontos da malha gravimétrica.

De posse dos dados gravimétricos corrigidos, foram geradas as anomalias Bouguer $\left(\mathrm{D}_{\mathrm{gB}}\right)$, subtraindo-se do valor da gravidade observado $\left(\mathrm{g}_{\mathrm{obs}}\right)$, já corrigido quanto aos efeitos de maré, à deriva instrumental e latitude, e ao valor da gravidade normal $\left(g_{n}\right)$, reduzido para o nível do terreno (calculado para a latitude de referência empregada na correção homônima), conforme a Equação 1:

$\mathrm{D}_{\mathrm{gB}}=\mathrm{g}_{\mathrm{obs}}-\mathrm{g}_{\mathrm{n}}+\mathrm{C}_{\mathrm{AL}}-\mathrm{C}_{\mathrm{B}}+\mathrm{C}_{\mathrm{T}}$

Onde:

Anomalias Bouguer $\left(\mathrm{D}_{\mathrm{gB}}\right)$;

Gravidade observado $\left(\mathrm{g}_{\mathrm{obs}}\right)$;

Gravidade normal $\left(\mathrm{g}_{\mathrm{n}}\right)$;

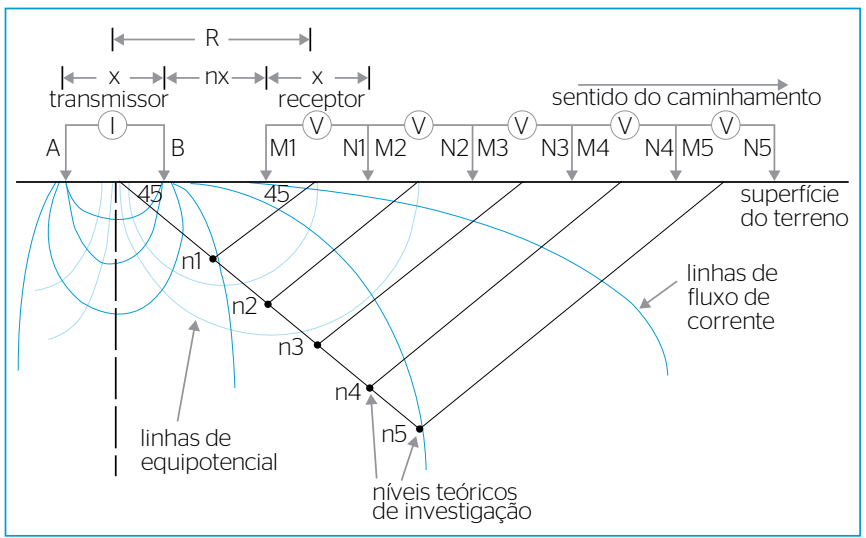

Figura 2 - Procedimento do caminhamento elétrico através do arranjo dipolo-dipolo, indicando os eletrodos de corrente (transmissor $A B$ ), de potencial (receptor $\mathrm{MN}$ ), linhas de equipotencial, de corrente e níveis teóricos de investigação.
Correção Ar Livre $\left(\mathrm{C}_{\mathrm{AL})}\right.$;

Correção Bouguer $\left(\mathrm{C}_{\mathrm{B}) \mathrm{e}}\right.$

Correção de Terreno $\left(\mathrm{C}_{\mathrm{T}}\right)$

As anomalias Bouguer, assim determinadas, são causadas pela presença e distribuição de massa entre a superfície física e a superfície de referência (geóide), o que permite, em um próximo passo, avaliar as dimensões (geometria, profundidade e densidade) dos corpos causadores das mencionadas anomalias. Em outras palavras, as anomalias Bouguer refletem os contrastes de densidade das rochas em subsuperfície.

\section{RESULTADOS E DISCUSSÃO}

\section{Gravimetria}

As Figuras 3A e 3B mostram os mapas das anomalias gravimétricas de Bouguer e Residuais. Observa-se que a porção oriental é caracterizada por sinais com maiores amplitudes, em contraposição ao segmento ocidental, onde as mesmas são intermediárias a baixas, destacando-se valores mínimos entre as estações 0-44 $\mathrm{m}$ das linhas L4, L5 e L6. A Figura 3B evidencia uma tendência central de resíduos negativos, disposto na direção aproximada N-S, caracterizando um trato de interesse, pois o mesmo revela contraste negativo de densidade dos prováveis alvos em relação ao meio envolvente.

\section{Resistividade elétrica}

Os resultados são apresentados em escala de cores e valores, evidenciando dessa forma os modelos de inversão (FACHIN et al., 2006; CORRÊA ALEGRIA et al., 2009) que representam variação do parâmetro resistividade elétrica, cujo comportamento da corrente elétrica está relacionado com o meio por onde passa (WILSON et al., 2006; GEORGAKI et al., 2008).

As Figuras $4 \mathrm{~A}$ a $4 \mathrm{M}$ exibem as pseudo-seções de resistividade real e os modelos de profundidade, representativos da distribuição lateral e vertical das resistividades verdadeiras, respectivamente das linhas L1 a L13. Como pode-se observar nas ilustrações, em função do espaçamento de $4 \mathrm{~m}$ entre os eletrodos de corrente $(\mathrm{AB})$ e de potencial $(\mathrm{MN})$, a profundidade máxima modelada é de $16 \mathrm{~m}$, sendo que os valores mais seguros situam-se da superfície até cerca de $8 \mathrm{~m}$, considerando-se que os demais $8 \mathrm{~m}$ são inferidos pelo processo de inversão utilizado para gerar os citados modelos de profundidade.

Outro aspecto que sobressai nas referidas figuras é o contraste de resistividade, sobretudo nos níveis inferiores a $5 \mathrm{~m}$ de profundidade, entre as porções sudeste das linhas L1 a L13, caracterizadas por valores menores que $300 \mathrm{ohm} . \mathrm{m}$, mais próximas do lago, e os tratos posicionados a noroeste, cujas magnitudes são maiores que 400 ohm.m. Tal contraste reflete o nível freático, mais condutivo, o qual é mais raso 


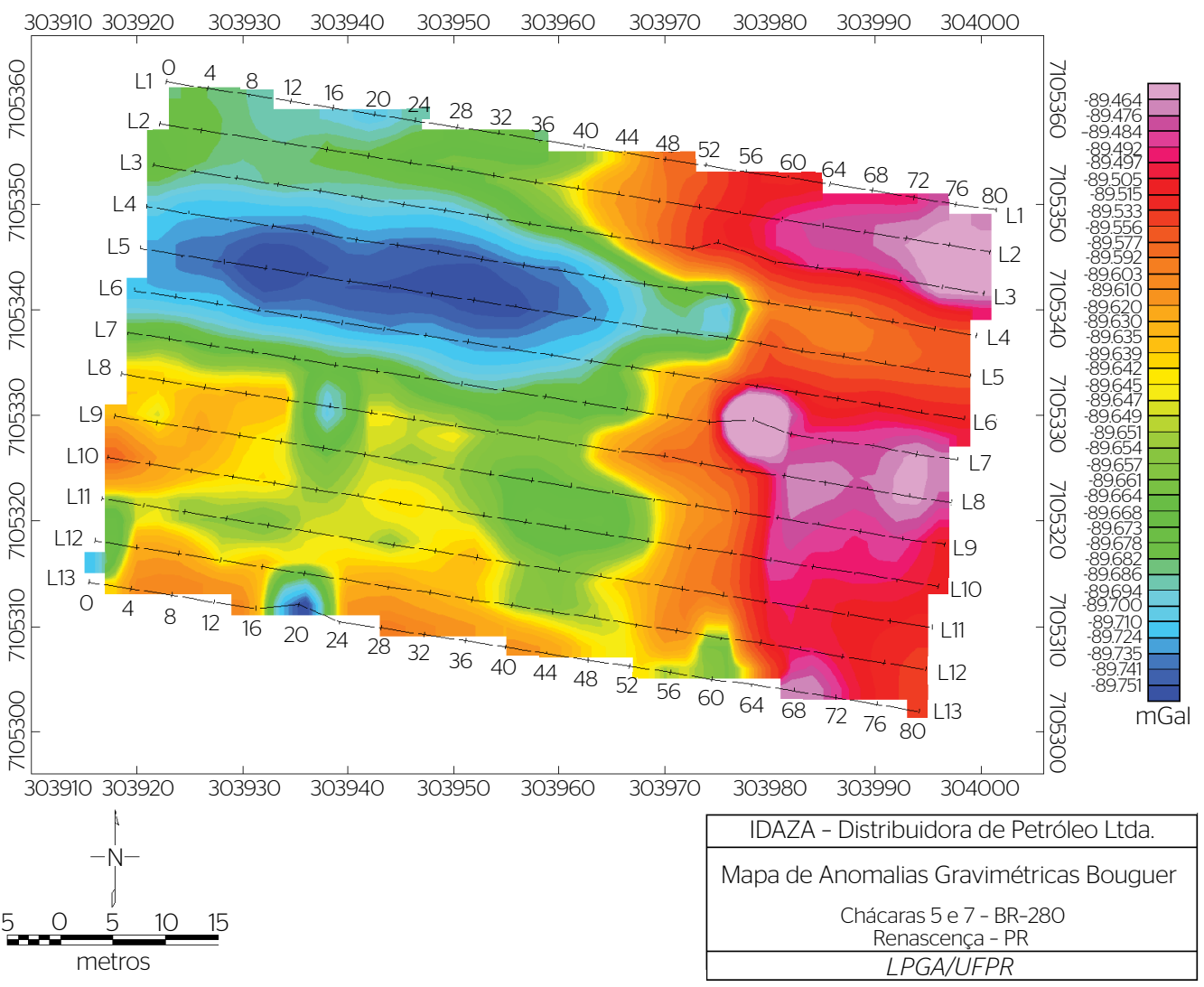

B

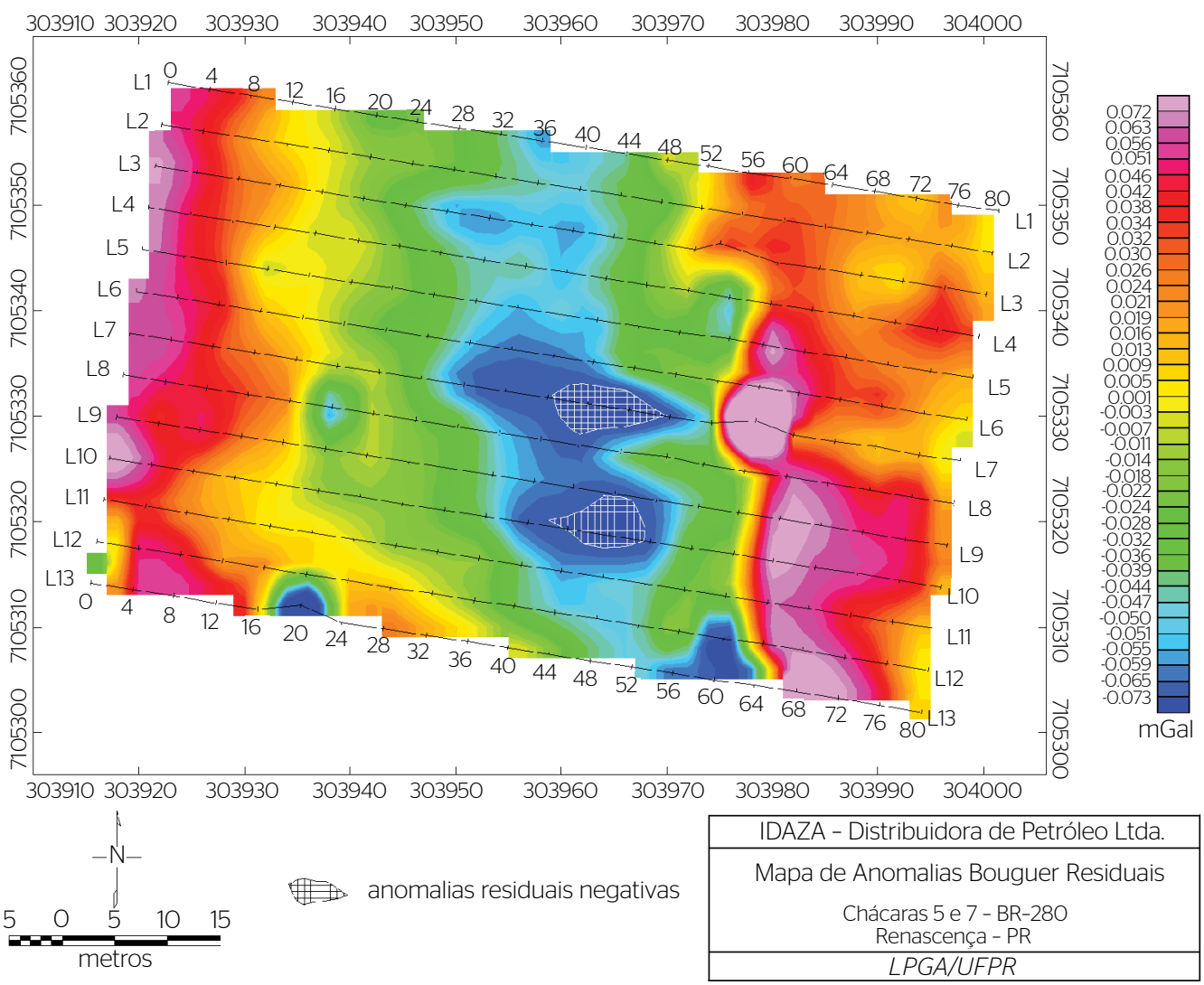

Figura 3 - (A) Distribuição espacial das anomalias gravimétricas. (B) Distribuição espacial das anomalias de Bourguer Residuais. 
nos tratos sudeste, em contraposição àqueles posicionados a noroeste, onde o nível da água é mais profundo, comportamento esse que guarda relação direta com a topografia da área estudada.

Entretanto, considerando o objetivo primordial da pesquisa, atenção especial deve ser dada às respostas dos níveis mais superficiais, a fim de verificar eventuais comportamentos anômalos da resistividade, os quais podem refletir, além da fonte, a dispersão/migração de hidrocarbonetos em subsuperfície, reveladas por elevados valores (aspecto quantitativo) e formas conspícuas (aspecto qualitativo); não obstante, como se verifica nas Figuras $4 \mathrm{~A}$ a $4 \mathrm{M}$, o padrão de distribuição lateral e vertical daquela propriedade física é muito semelhante.

O exame detalhado das figuras retromencionadas, no contexto da perspectiva anteriormente descrita, apontou anomalias (ORLANDO \& MARCHESI, 2001; PORSANI et al., 2004) como aquelas características nas linhas L8, L9 e L10, conforme indicado nas Figuras 4H, 4I e 4J. Trabalhos como os de Braga et al. (2005), Moreira et al. (2006) e Lago et al. (2009), desenvolvidos em áreas contaminadas por hidrocarbonetos, também encontraram valores altos de resistividade em função do meio resistivo/condutivo. É interessante notar que uma característica comum a tais anomalias, além dos elevados índices da resistividade verdadeira e de suas formas singulares, é a presença de pequenas perturbações condutivas rasas (máximo de $1 \mathrm{~m}$ ), as quais devem refletir coberturas argilosas e saturadas não contaminadas.

$\mathrm{Na}$ Figura $4 \mathrm{H}$, além da forma conspícua, a resistividade verdadeira da linha L8 é superior a 620 ohm.m, entre as estações 48 e 68 m, atingindo uma profundidade máxima de $4 \mathrm{~m}$ abaixo da superfície do terreno, em torno da estação $56 \mathrm{~m}$. Na Figura 4I, apesar dos valores de resistividade da linha L9 não serem anômalos (superiores a 578 ohm.m), sua forma chama a atenção entre as estações 48 e $64 \mathrm{~m}$, com profundidade máxima de $2 \mathrm{~m}$. Na Figura 4J, entre as estações 20 e $40 \mathrm{~m}$ da linha L10, assinala-se a mais expressiva anomalia de resistividade real, considerando sua forma ímpar, valores de resistividade real superiores a $1.000 \mathrm{ohm} . \mathrm{m}$ e profundidade máxima de cerca de $7 \mathrm{~m}$, centrada na estação $30 \mathrm{~m}$.

A fim de avaliar quantitativamente, com mais precisão, os valores de resistividade real dos modelos de profundidade, foi construída a Tabela 1, que mostra os valores mínimos, máximos, as médias e os desvios padrão de cada linha levantada. É perceptível que na linha L10 se assinale o maior índice encontrado (46.655 ohm.m), assim como a média mais elevada (1.009 ohm.m), seguido da linha L8 (máximo de 12.763 ohm.m, com média de 505 ohm.m). De fato, os valores de resistividade real da linha $\mathrm{L} 9$, como já referido, não são significativos (máximo de 1.372 ohm.m), com média de 386 ohm.m, tendo sido selecionada para verificação direta tão-somente pela sua forma. Na referida tabela, também são exibidas, para efeito de comparação, as médias dos valores mínimos (35 ohm.m), máximos (2.276 ohm.m), das médias (432 ohm.m) e dos desvios padrão (344 ohm.m) de todas as linhas levantadas e invertidas (L1 a L13), com exclusão daqueles correspondentes às linhas L8 e L10.

Na Figura 5, evidenciam-se os locais anômalos derivados da interpretação dos resultados gravimétricos e resistivimétricos, sendo esta última sobre o modelo digital de elevação (MDE), as quais indicam os pontos, por ordem de prioridade, que devem ser objeto de verificações diretas.

Após as interpretações dos dados da resistividade elétrica e gravimétricos, elencaram-se quatro áreas para as prospecções.

Com relação ao ponto 1 (Figura 5), esse representa a anomalia de resistividade centrada na estação $30 \mathrm{~m}$. A escavação nesse local revelou fragmentos de uma laje de concreto armado, responsável pela referida anomalia com altos valores de resistividade, conforme a Tabela 1.

No que se refere aos pontos 2, 3, 4 e 5 da Figura 5, ressalta-se que os pontos 2 e 3 estão relacionados com anomalias de

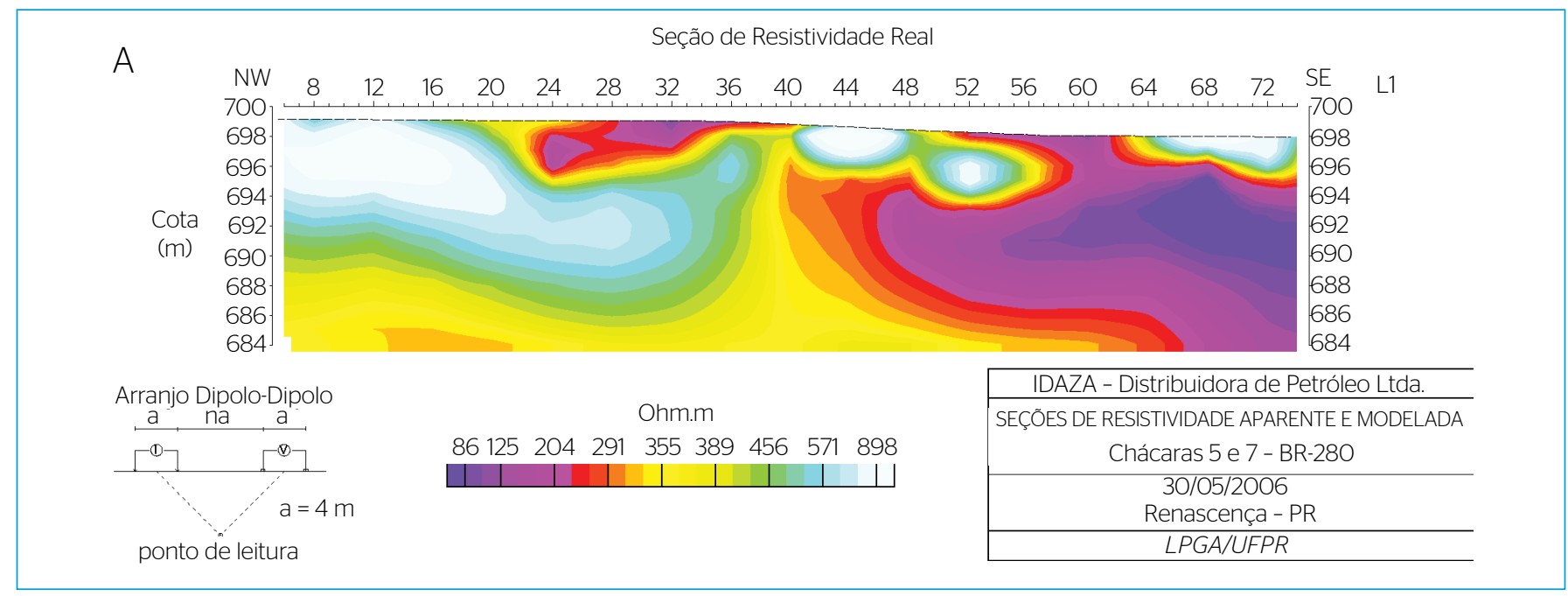

Figura 4 - Seções das resistividades reais das 13 linhas pesquisadas. 
Seção de Resistividade Real

B

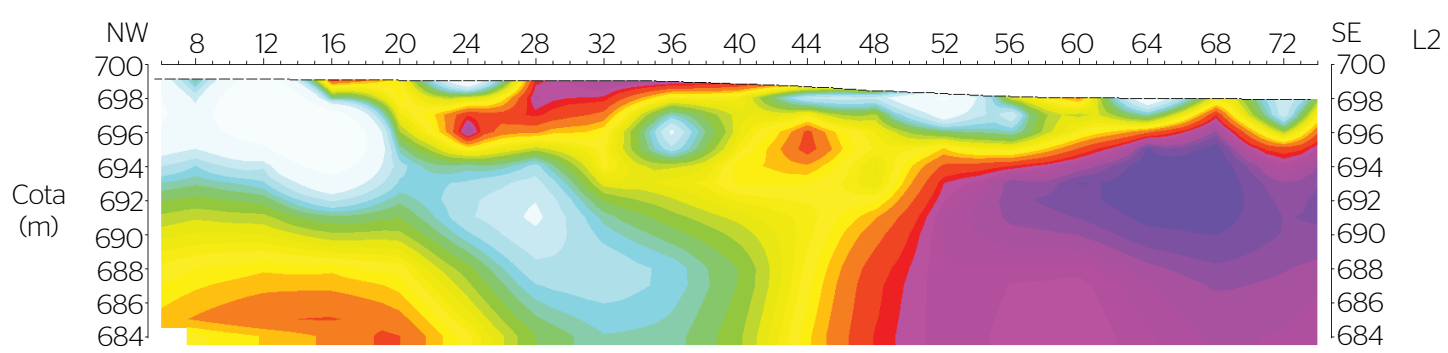

Arranjo Dipolo-Dipolo

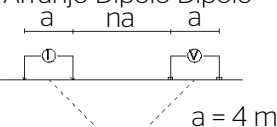

ponto dé leitura
Ohm.m

$91134 \quad 184 \quad 253 \quad 329 \quad 386 \quad 462 \quad 552 \quad 849$
IDAZA - Distribuidora de Petróleo Ltda SEÇÕES DE RESISTIVIDADE APARENTE E MODELADA Chácaras 5 e 7 - BR-280 $02 / 06 / 2006$ Renascença - PR $\angle P G A / U F P R$

Seção de Resistividade Real

C

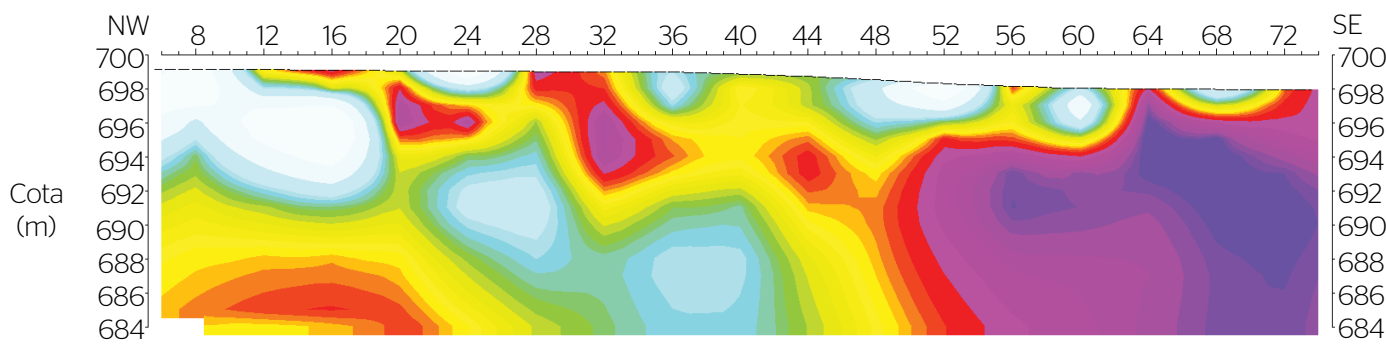

Arranjo Dipolo-Dipolo a, na, a $\begin{array}{ll}{[\mathbb{1}]} & \square] \\ \ddots & a=4 \mathrm{~m}\end{array}$

ponto de leitura
Ohm.m

$84113 \quad 191 \quad 282336 \quad 393 \quad 464534901$
IDAZA - Distribuidora de Petróleo Ltda SEÇÕES DE RESISTIVIDADE APARENTE E MODELADA Chácaras 5 e 7 - BR-280 30/05/2006 Renascença - PR LPGA/UFPR

Seção de Resistividade Real

D

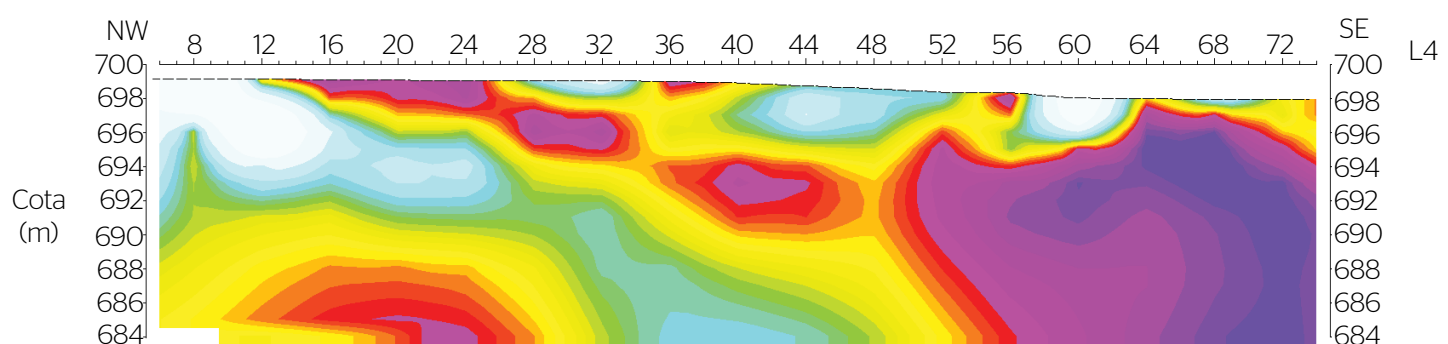

Arranjo Dipolo-Dipolo

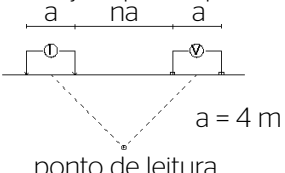

Ohm.m

$79119209282 \quad 346 \quad 406468549970$

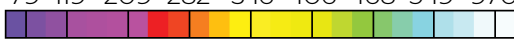

IDAZA - Distribuidora de Petróleo Ltda. SEÇÕES DE RESISTIVIDADE APARENTE E MODELADA Chácaras 5 e 7 - BR-280 01/06/2006 Renascença - PR 
Seção de Resistividade Real

E

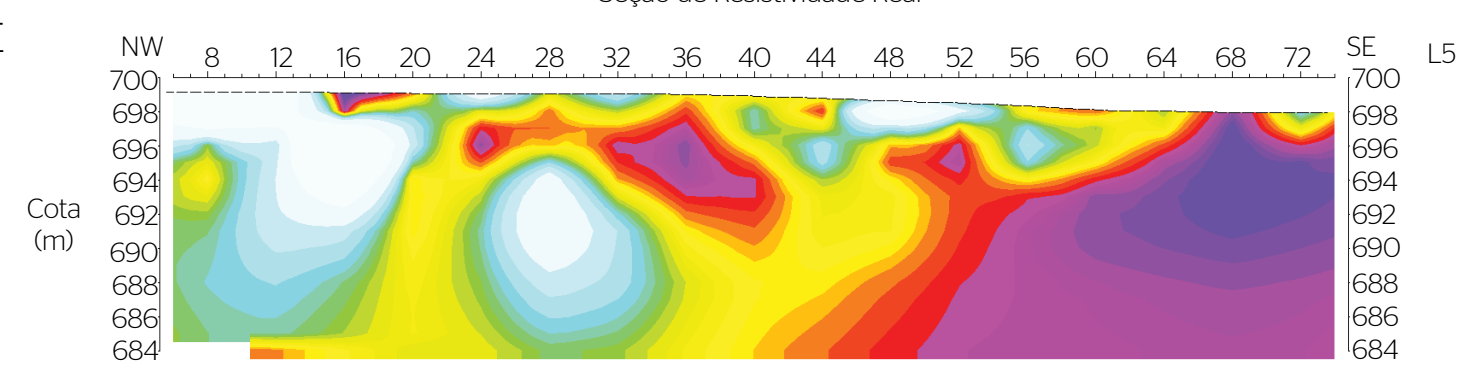

Arranjo Dipolo-Dipolo \begin{tabular}{cc} 
a & na \\
\hdashline$-a$ & $a$ \\
$\ddots$ & $a=4 \mathrm{~m}$
\end{tabular}

ponto de leitura
Ohm.m

$98143 \quad 183 \quad 263 \quad 331 \quad 391 \quad 454539905$

\begin{tabular}{c}
\hline IDAZA - Distribuidora de Petróleo Ltda. \\
\hline $\begin{array}{c}\text { SEÇÕES DE RESISTIVIDADE APARENTE E MODELADA } \\
\text { Chácaras } 5 \text { e } 7 \text { - BR-280 }\end{array}$ \\
\hline $\begin{array}{c}\text { 31/O5/2006 } \\
\text { Renascença - PR }\end{array}$ \\
\hline LPGA/UFPR
\end{tabular}

Seção de Resistividade Real

F

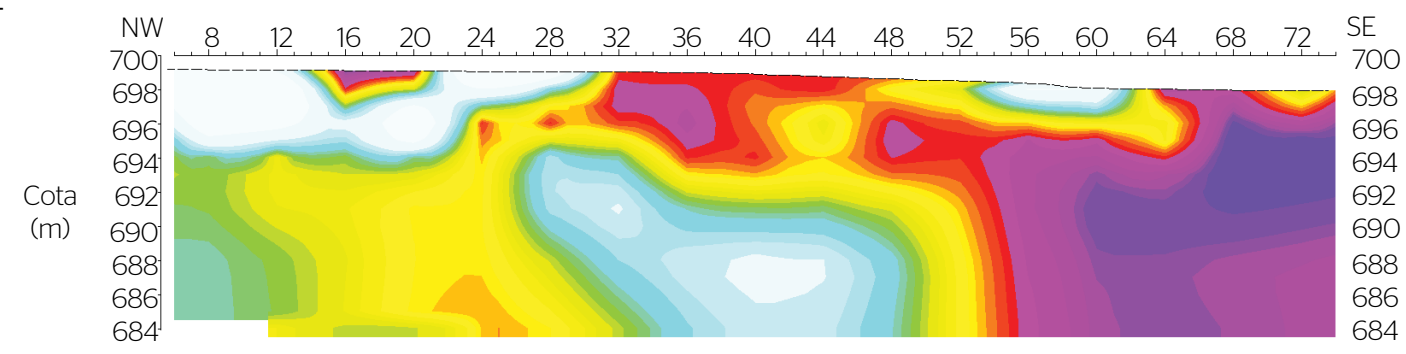

698

696

694

692

688 684

Arranjo Dipolo-Dipolo

\begin{tabular}{cc} 
a, na & a \\
\hdashline-1 & $\square$ \\
$\ddots$ & $a=4 m$
\end{tabular}

Ohm.m

$\begin{array}{llllllll}108 & 142 & 217 & 280 & 342 & 406 & 465 & 561\end{array}$

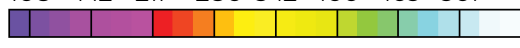

IDAZA - Distribuidora de Petróleo Ltda. SEÇÕES DE RESISTIVIDADE APARENTE E MODELADA Chácaras 5 e 7 - BR-280

ponto de leitura

01/06/2006

Renascença - PR $\angle P G A / U F P R$

Seção de Resistividade Real

G

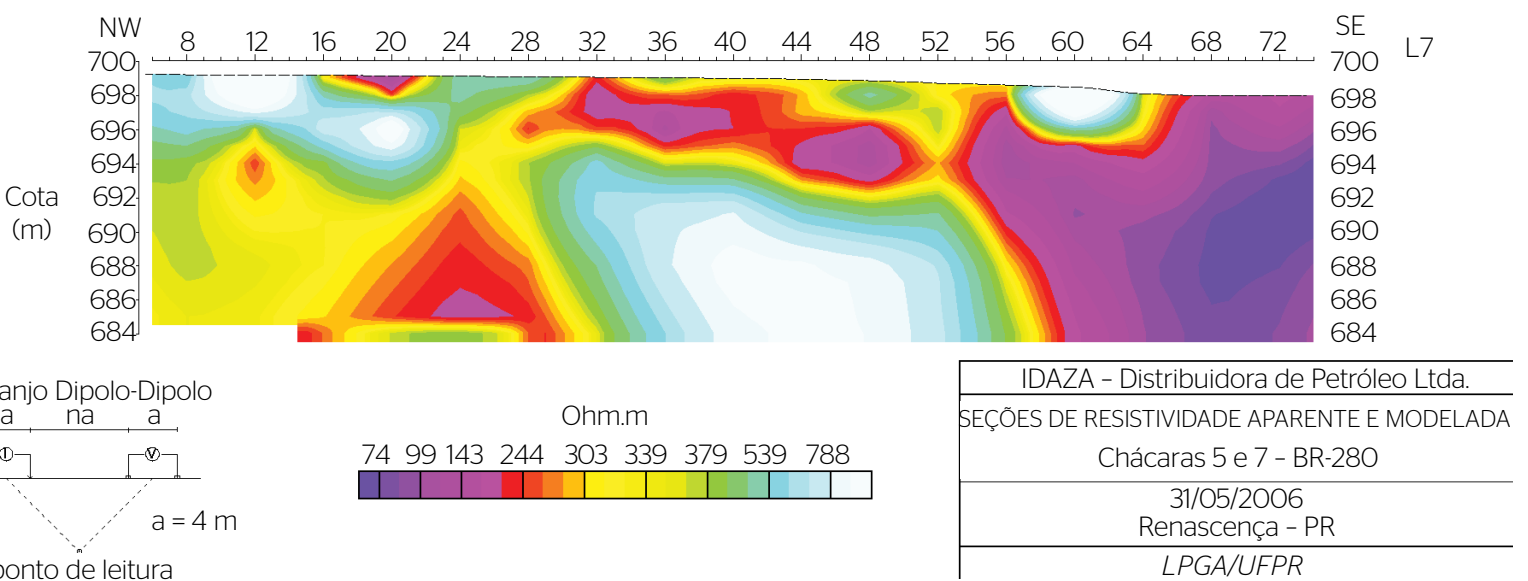


$\mathrm{H}$

Seção de Resistividade Real
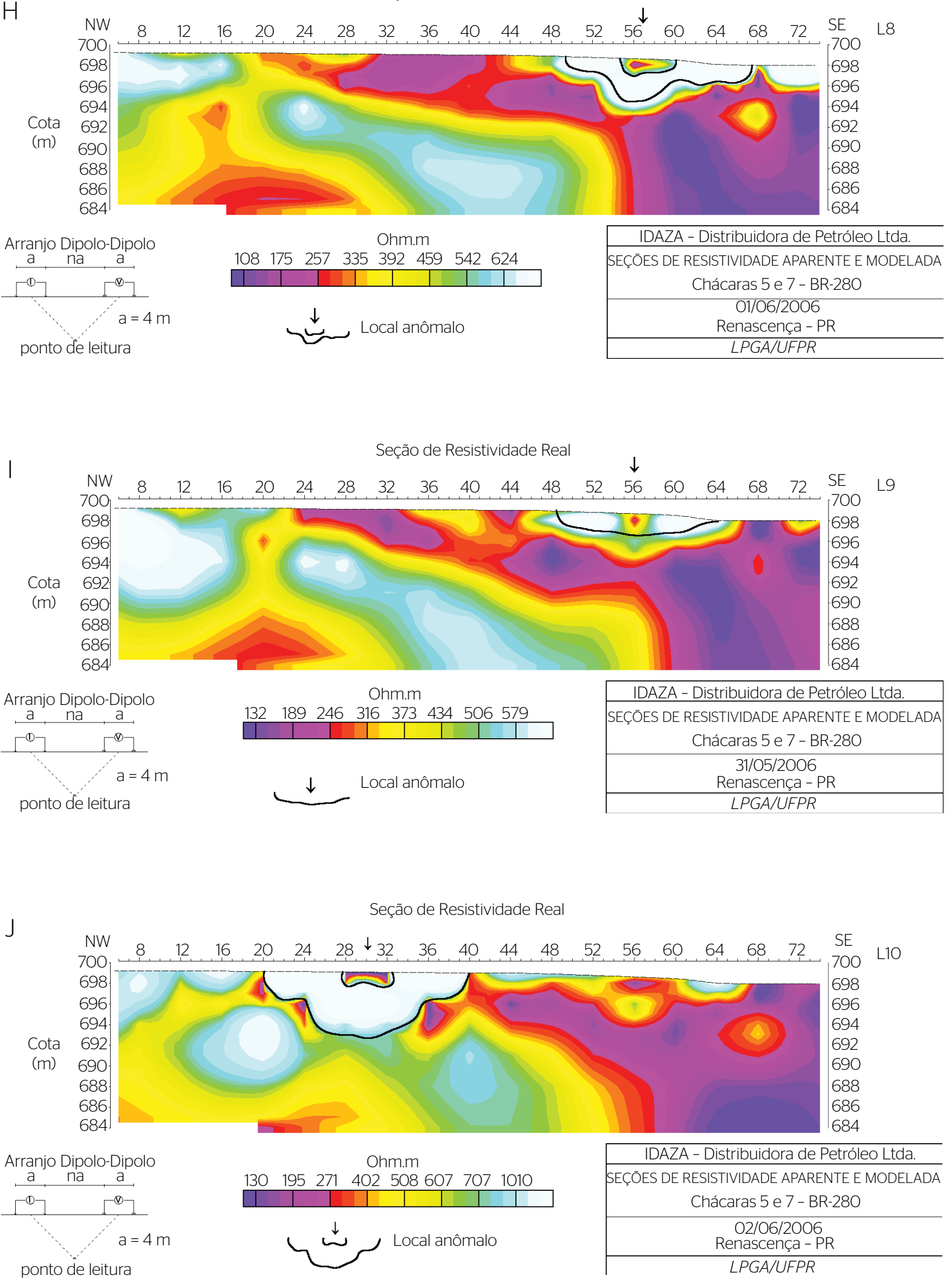
K

Seção de Resistividade Real

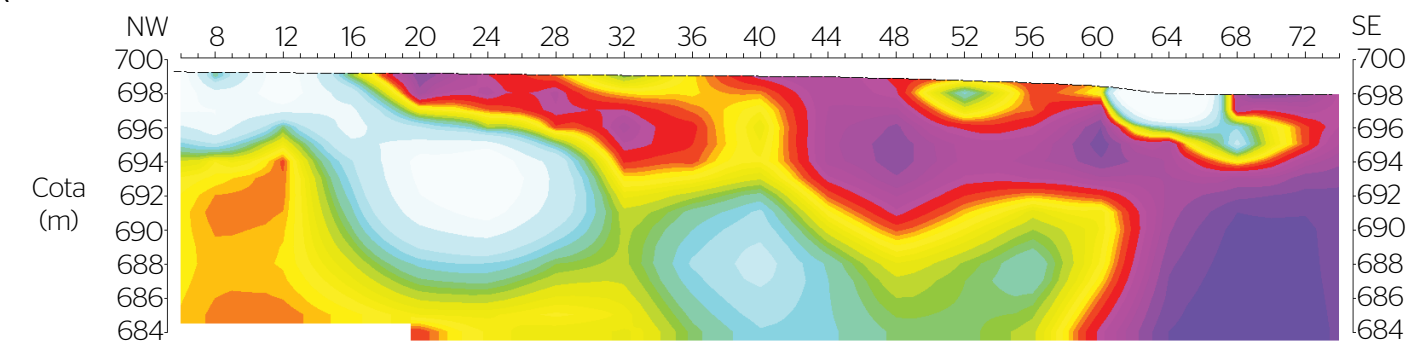

L11

Arranjo Dipolo-Dipolo

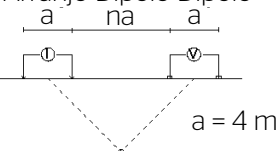

Ohm.m

ponto de leitura

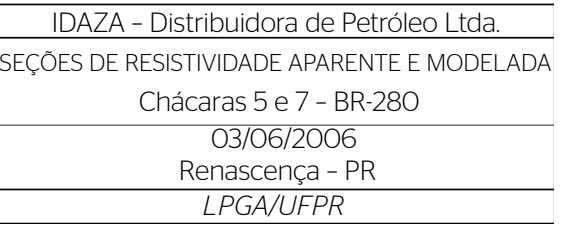

L

Renascença - PR

Seção de Resistividade Real

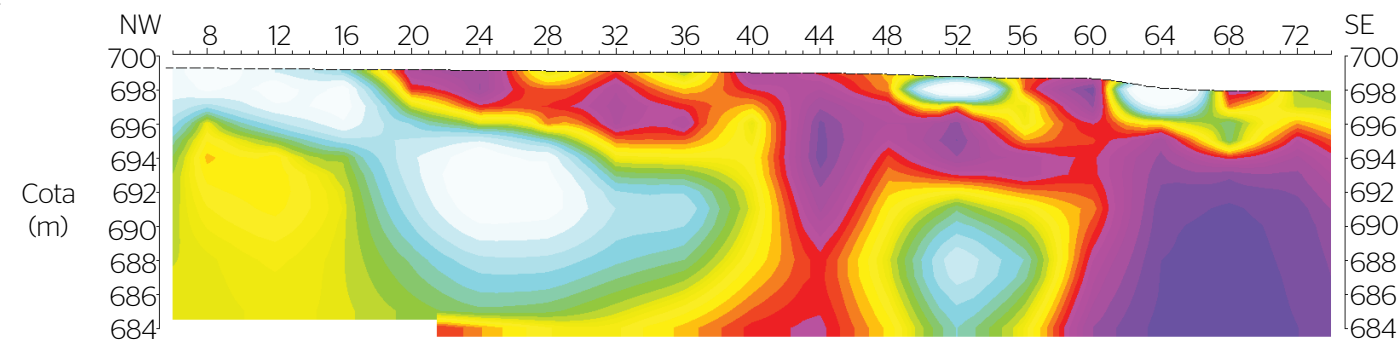

Arranjo Dipolo-Dipolo

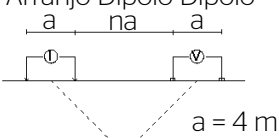

ponto de leitura
Ohm.m

$56160289386495 \quad 572 \quad 666 \quad 8281150$
IDAZA - Distribuidora de Petróleo Ltda. SEÇÕES DE RESISTIVIDADE APARENTE E MODELADA Chácaras 5 e 7 - BR-280 03/06/2006 Renascença - PR

M

Seção de Resistividade Real

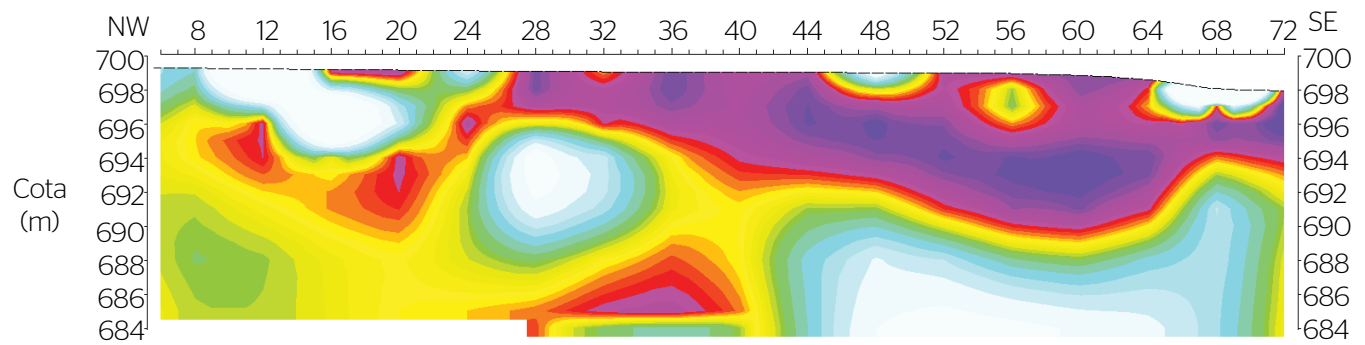

Arranjo Dipolo-Dipolo

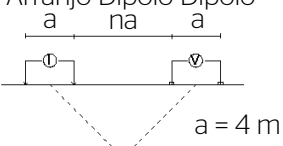

ponto de leitura
Ohm.m

$\begin{array}{llllllll}157 & 297 & 422 & 532 & 601 & 681 & 802 & 1043\end{array}$

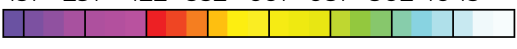

IDAZA - Distribuidora de Petróleo Ltda. SEÇÕES DE RESISTIVIDADE APARENTE E MODELADA Chácaras 5 e 7 - BR-280 O3/06/2006
Renascença - PR $\angle P G A / U F P R$ 
resistividade das linhas L8 e L9, enquanto os pontos 4 e 5 representam anomalias gravimétricas residuais negativas. A verificação direta dos referidos pontos resultou em fragmentos de rocha basáltica não alterada, envolvidos por solos, explicando os altos valores de resistividade dos pontos 2 e 3 indicados na Tabela 1, enquanto as anomalias gravimétricas dos pontos 4 e 5 poderiam ser explicadas pelo contraste negativo de densidade entre os solos e os fragmentos rochosos.

A interface resistiva/condutiva, observada nas linhas L8 e L9, estações 64 e $68 \mathrm{~m}$, assim como um núcleo medianamente resistivo envolvido por uma zona condutiva centrada na estação $68 \mathrm{~m}$ motivaram a verificação direta dessas anomalias. Após escavados $2 \mathrm{~m}$ de profundidade (Figura 6), sentiu-se um forte odor de hidrocarboneto que

Tabela 1 - Estatísticas descritivas dos valores de resistividade real (ohm.m) das linhas L1 a L13.

\begin{tabular}{l|c|c|c|c} 
N=147 & Mínimo & Máximo & Média & Desvio padrão \\
\hline Linha L1 & 35 & 1.976 & 403 & 295 \\
\hline Linha L2 & 51 & 1.574 & 390 & 275 \\
\hline Linha L3 & 44 & 1.455 & 392 & 285 \\
\hline Linha L4 & 25 & 2.302 & 435 & 395 \\
\hline Linha L5 & 37 & 2.307 & 396 & 344 \\
\hline Linha L6 & 58 & 1.310 & 356 & 251 \\
\hline Linha L7 & 51 & 1.812 & 360 & 294 \\
\hline Linha L8 & 16 & 12.763 & 505 & 1.107 \\
\hline Linha L9 & 31 & 1.372 & 386 & 245 \\
\hline Linha L10 & 11 & 46.655 & 1.009 & 4.064 \\
\hline Linha L11 & 34 & 3.288 & 467 & 389 \\
\hline Linha L12 & 20 & 2.842 & 549 & 423 \\
\hline Linha L13 & 03 & 4.797 & 616 & 590 \\
\hline Média* & 35 & 2.276 & 432 & 344 \\
\hline
\end{tabular}

*Médias de resistividade real (ohm.m), calculadas com exclusão dos valores das linhas L8 e L10.

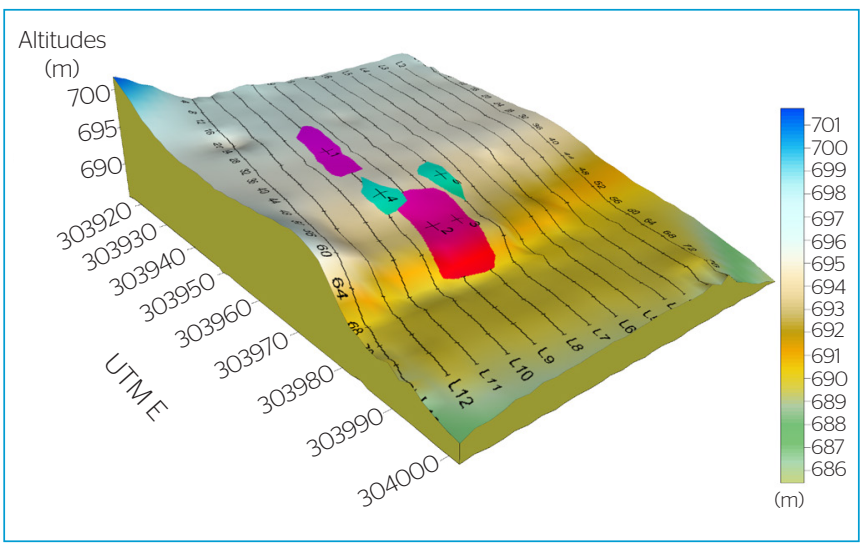

Figura 5 - Mapa integrado de interpretação geofísica sobre o Modelo Digital de Elevação. impregnava todo o perfil do solo até atingir a profundidade de $3 \mathrm{~m}$ no nível do lençol freático, evidenciando assim a contaminação da área.

Foram realizadas amostragens de solos e água que, após análises por cromatografia gasosa, revelaram concentrações médias de $536 \mathrm{mg} . \mathrm{L}^{-1}$ em solos e $618 \mathrm{mg} / \mathrm{l}$ em água de hidrocarbonetos poli-aromáticos (HPAs). Tomando-se por base a resolução CONAMA n 357/2005, que estabelece "Condições e Padrões de Lançamento de Efluentes", o limite de óleos minerais é de até $20 \mathrm{mg}$. $\mathrm{L}^{-1}$. As amostras ultrapassaram em mais de 26 a 30 vezes, respectivamente, o limite máximo permitido.

\section{CONCLUSÃO}

A utilização do método geofísico da resistividade elétrica foi fundamental para a descoberta da célula contaminada com hidrocarboneto.

Detectou-se a presença de hidrocarboneto a $3 \mathrm{~m}$ de profundidade nas linhas L8 e L9, especificamente nas estações 64 e 68.

As altas concentrações de HPAs evidenciam forte contaminação do passivo ambiental, objeto da presente pesquisa.

A remediação da área deverá ser feita com a remoção dos solos contaminados para tratamento in situ ou ex situ. Caso não sejam tratados, os solos retirados deverão ser depositados em aterro industrial.

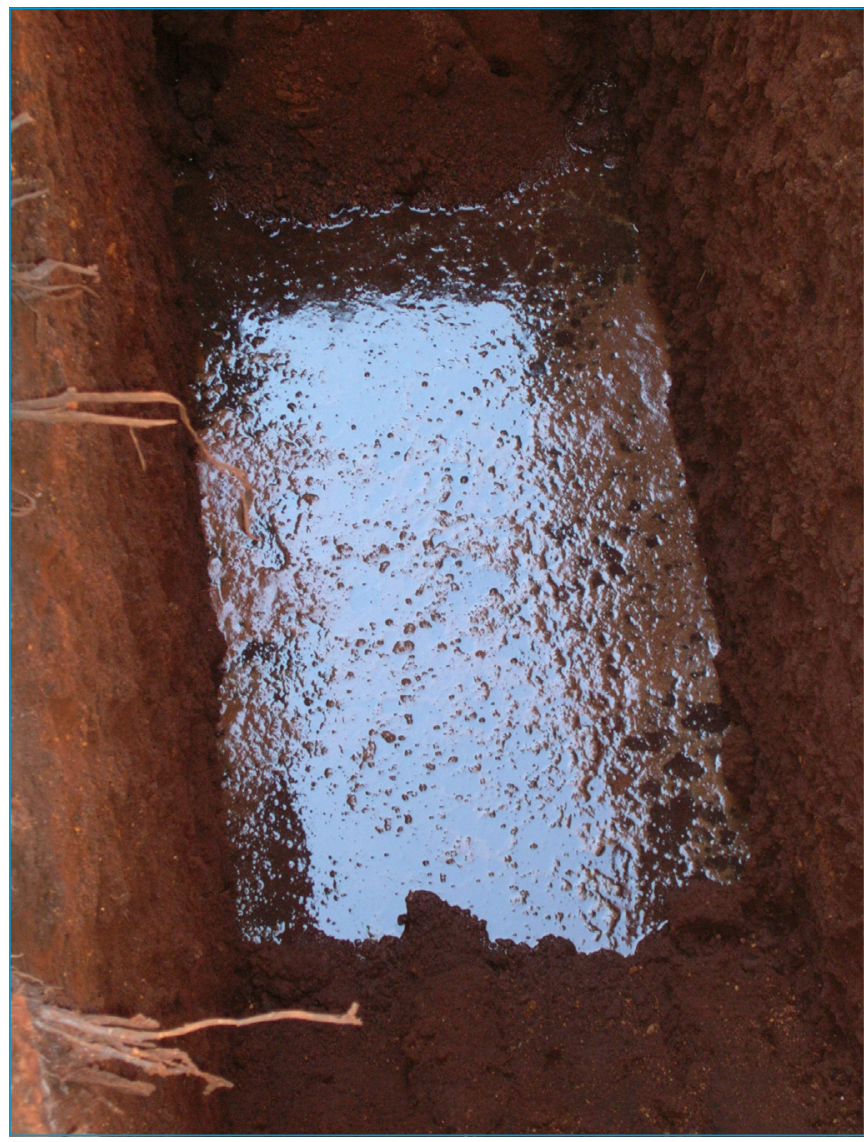

Figura 6 - Escavação com surgimento do lençol freático e pluma de contaminação. 


\section{REFERÊNCIAS}

Amato, M.; Bitella, G.; Rossi, R.; Gómez, J.A.; Lovelli, S.; Gomes, J.J.F. (2009) Multi-electrode 3D resistivity imaging of alfalfa root zone. European Journal of Agronomy, v. 31, n. 4, p. 213-222.

Atekwana, E.A.; Sauck, W.A.; Werkema Jr., D.D. (2000) Investigations of geoelectrical signatures at a hydrocarbon contaminated site. Journal of Applied Geophysics, v. 44, n. 2-3, p. 167-180.

BECEGATO, V.A.; FERREIRA, F.J.F. (2005) Gamaespectrometria, resistividade elétrica e susceptibilidade magnética de solos agrícolas no noroeste do estado do Paraná. Revista Brasileira de Geofísica, v. 23, n. 4, p. 371-405.

BECEGATO, V.A.; STEVANATO, R.; FERREIRA, F.J.F.; CUNHA, J.U.; CABRAL, J.B.P. (2009) Investigações geofísicas aplicadas no lixão desativado do município de Saudades do Iguaçú-PR. Revista Geográfica Acadêmica, v. 3, n. 1, p. 47-55.

BERNSTONE, C:; DAHLIN, T.; OHLSSON, T.; HOGLAND, W. (2OOO) DC-resistivity mapping of internal landfill structures: two preexcavation surveys. Environmental Geology, v. 39, n. 3-4, p. 360-371.

BERTOLLA, L.; PORSANI, J.L.; SOLDOVIERI, F.; CATAPANO, I. (2014) GPR-4D monitoring a controlled LNAPL spill in a masonry tank at USP, Brazil. Journal of Applied Geophysics, v. 103, p. 237-244.

BORTOLOZO, C.A.; COUTO JR., M.A.; PORSANI, J.L.; ALMEIDA, E.R.; MONTEIRO SANTOS, F.A. (2014) Geoelectrical characterization using joint inversion of VES/TEM data: A case study in Paraná Sedimentary Basin, São Paulo State, Brazil. Journal of Applied Geophysics, v. 111, p. 33-46

BRAGA, A.C.; CARDINALI, M.T. (2005) Aplicação da resistividade e cargabilidade em estudos de contaminação de sedimentos por derivados de hidrocarbonetos. Revista Brasileira de Geofísica, v. 23, n. 2, p. 181-190

CORREAA ALEGRIA, F.; MARTINHO, E.; ALMEIDA, F. (2009) Measuring soil contamination with the time domain induced polarization method using LabVIEW. Measurement, v. 42, п. 7 , p. 1082-1091.

de la Vega, M.; Osella, A.; Lascano, E. (2003) Joint inversion of Wenner and dipole - dipole data to study a gasoline-contaminated soil. Journal of Applied Geophysics, v. 54, n. 1-2, p. 97-109.

FACHIN, S.J.S.; HUBER, F.; SHIRAIWA, S.; BORGES W.R.; MIGLIORINI, R.B. (2006) Aplicação de métodos geofísicos para identificar áreas contaminadas por resíduos de um curtume. Revista Brasileira de Geofísica, v. 24, n. 1, p. 129-138.

Friedel, S.; Thielen, A.; Springman, S.M. (2006) Investigation of a slope endangered by rainfall-induced landslides using 3D resistivity tomography and geotechnical testing. Journal of Applied Geophysics, v. 60, n. 2, p. 100-114.

GEORGAKI, I.; SOUPIOS, P.; SAKKAS, N.; VERVERIDIS, F.; TRANTAS, E.; VALLIANATOS, F.; MANIOS, T. (2008) Evaluating the use of electrical resistivity imaging technique for improving $\mathrm{CH} 4$ and CO2 emission rate estimations in landfills. Science of the Total Environmental, v. 389, n. 2-3, p. 522-531.

HADZICK, Z.Z:; GUBER, A.K.; PACHEPSKY, Y.A.; HILL, R.L. (2O11) Pedotransfer functions in soil electrical resistivity estimation. Geoderma, v. 164, n. 3-4, p. 195-202.

LAGO, A.L.; Elis, V.R.; Borges, W.R.; Penner, G.C. (2009) Geophysical investigation using resistivity and GPR methods: a case study of a lubricant oil waste disposal area in the city of Ribeirão Preto, São Paulo, Brazil. Environmental Geology, v. 58, n. 2, p. 407-417.

Martinho, E.; Almeida, F.; Senos Matias, M.J. (2006) An experimental study of organic pollutant effects on time domain induced polarization measurements. Journal of Applied Geophysics, v. 60, p. 27-40.

MENEZES, A.M.; MOREIRA, C.A.;ILHA, L.M.; SCHWEIG, C. (2O11) Estudo geofísico de vazamento de combustíveis em posto de abastecimento. Geociências, v. 30, n. 4, p. 601-609.

MOREIRA, C.A.; DOURADO, J.C.; BRAGA, A.C.O. (2006) Aplicação de técnica de caminhamento elétrico em área contaminada por derivados de petróleo. Revista Brasileira de Geofísica, v. 24, n. 3 , p. 383-392.

Orlando, L.; Marchesi, E. (2001) Georadar as a tool to identify and characterise solid waste dump deposits. Journal of Applied Geophysics, v. 48, n. 3, p. 163-174.

PORSANI, J.L.; FILHO, W.M.; ELIS, V.R., SHIMELES, F.; DOURADO, J.C.; MOURA, H.P. (2004) The use of GPR and VES in delineating a contamination plume in a landfill site: a case study in SE Brazil. Journal of Applied Geophysics, v. 55, n. 3-4, p. 199-209.

ROSALES, R.M.; Martínez-Pagan, P.; Faz, A.; moreno-Cornejo, J. (2012) Environmental Monitoring Using Resistivity Electrical (ERT) in Subsoil of Three Former Petrol Stations in SE of Spain. Water, Air, \& Soil Pollution, v. 223, n. 7, p. 3757-3773.

Samouëlian, A.; Cousin, I., Tabbagh, A.; Bruand, A.; Richard, G. (2005) Electrical resistivity survey in soil science: a review. Soil \& Tillage Research, v. 83, n. 2, p. 173-193.

SAUCK, W.A. (2000) A model for the resistivity structure of LNAPL plumes and their environs in sandy sediments. Journal of Applied Geophysics, v. 44, n. 2-3, p. 151-165.

Séger, M.; Cousin, I.; Frison, A.; Boizard, H.; Richard, G. (2009) Characterisation of the structural heterogeneity of the soil tilled layer by using in situ 2D and 3D electrical resistivity measurements. Soil \& Tillage Research, v. 103, n. 2, p. 387-398 
Sudha, K.; Israil, M.; Mittal, S.; Rai, J. (2009) Soil characterization using electrical resistivity tomography and geotechnical investigations. Journal of Applied Geophysics, v. 67, n. 1, p. 74-79.

Ustra, A.T.; Elis, V.R.; Mondelli, G.; Zuquette, L.V.; Giacheti, H.L. (2012) Case study: a 3D resistivity and induced polarization imaging from downstream a waste disposal site in Brazil. Environmental Earth Sciences, v. 66, n. 3, p. 763-772.
WILSON, S.R.; INGHAM, M.; McCONCHIE, J.A. (2006) The applicability of earth resistivity methods for saline interface definition. Journal of Hidrology, v. 316, n. 1-4, p. 301- 312.

YOON, G.L.; PARK, J.B. (2001) Sensitivity of leachate and fine contents on electrical resistivity variations of sandy soils. Journal of Hazardous Materials, v. 84, n. 2-3, p. 147-161. 